\title{
Erprobung eines neuartigen akustischen Sensorsystems zur Bestimmung von Gasgehaltsverteilungen in Mehrphasensystemen
}

\author{
Michael Elfering ${ }^{1,2}$, Sven Annas ${ }^{1}$, Hans-Arno Jantzen ${ }^{1}$ und Uwe Janoske ${ }^{2}$ \\ ${ }^{1}$ Labor für Strömungstechnik und -simulation, FH Münster, Steinfurt, Deutschland \\ ${ }^{2}$ Lehrstuhl für Strömungsmechanik, Bergische Universität Wuppertal, Wuppertal, Deutschland \\ Kontakt: michael.elfering@fh-muenster.de
}

\begin{abstract}
Mischvorgänge in Mehrphasenströmungen mit Gasanteil haben eine besondere Relevanz in der Verfahrenstechnik und treten in einer Vielzahl verschiedener Prozesse auf. Hierbei ist der Gasgehalt, das Verhältnis des Gasvolumens zum Gesamtvolumen, ein wichtiger Parameter bei der Auslegung und Bewertung der Durchmischung in Reaktoren. Als Messgröße ermöglicht die Gasgehaltsverteilung einen Einblick in die zugrundeliegenden Prozesse und deren räumliche Abläufe, sodass Verfahren zuverlässig überwacht und Optimierungsmaßnahmen der Anlagentechnik zielgerichtet durchgeführt werden können.

Die ortsaufgelöste Erfassung und Beschreibung dieser Größe ist Gegenstand aktueller Forschung. Neueste Ansätze von Elfering et al. [1, 2] forcieren die Entwicklung von Sensorsystemen zur Bestimmung des Gasgehalts in Suspensionen mittels akustischer Transmissionstomographie (GHATT). GHATT nutzt die Abhängigkeit der Schallgeschwindigkeit $c_{m}$ niederfrequenter Signale vom volumetrischen Gasgehalt $\varepsilon$ (Abb. 1). Mit Hilfe von Laufzeitmessungen wird bei der GHATT zunächst die Schallgeschwindigkeitsverteilung ermittelt, aus welcher anschließend der Gasgehalt ortsaufgelöst bestimmt wird. Die Abhängigkeit von Schallgeschwindigkeit und Gasgehalt wurde erstmals von Wood [3] mathematisch beschrieben und gilt für den sogenannten unterkritischen Frequenzbereich, d.h. unterhalb der Resonanzfrequenz für radiale Pulsationen der Blasen [4].
\end{abstract}

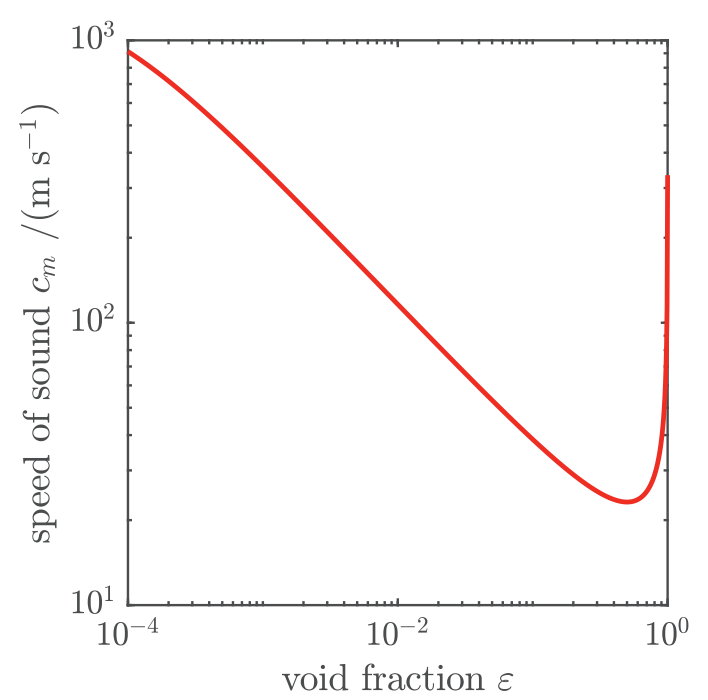

Abb. 1: Schallgeschwindigkeit $c_{m}$ niederfrequenter Signale in Wasser-Luft-Gemisch in Abhängigkeit vom volumetrischen Gasgehalt $\varepsilon$
Für die Laufzeitmessung bei der GHATT stellt die Resonanzfrequenz der Blasen somit eine natürliche obere Frequenzgrenze für die zu verwendenden Signale dar; Frequenzen oberhalb dieser Grenzen lassen sich nicht für die GHATT einsetzen. Die Signalgestaltung und -auswertung muss entsprechend an dieses Verfahren angepasst werden. Vor diesem Hintergrund thematisiert diese Arbeit die Entwicklung und Erprobung der GHATT und deren Signalauswertung.

\section{GHATT-Verfahren}

Für die tomographische Bestimmung des Gasgehaltes werden bei der GHATT akustische Sender und Empfänger im Messvolumen positioniert (Abb. 2). Aufeinander folgend wird das Volumen von den Sendern durchschallt und die Signale werden von den Empfängern aufgezeichnet.

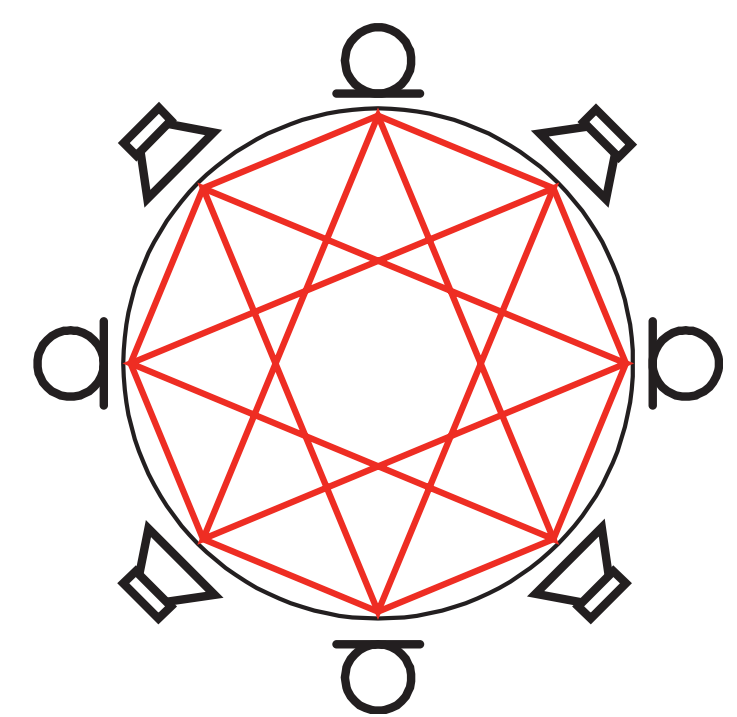

Abb. 2: Aufbau des Messsystems - schematisch dargestellt mit jeweils vier Sendern und Empfängern sowie den individuellen Messstrecken (rot)

Für jede dieser Messstrecken wird die individuelle Laufzeit ermittelt und im nächsten Schritt auf Basis der Laufzeiten ein Schnittbild der Schallgeschwindigkeitsverteilung tomographisch rekonstruiert. Da die lokale Schallgeschwindigkeit bei geeigneter Signalfrequenz primär vom lokalen Gasgehalt abhängt [5], lässt sich hieraus die Verteilung des Gasgehaltes ermitteln. 


\section{Laufzeitbestimmung}

Die Laufzeitbestimmung der GHATT setzt prinzipiell die Kenntnis der gesendeten und empfangenen Signale voraus. Unter Idealbedingungen entspricht das Empfangssignal $x$ dem um $\tau$ zeitverzögerten und um $\alpha$ gedämpften Sendesignal $s$ :

$$
x(t)=\alpha \cdot s(t-\tau)
$$

Bei Messungen in geschlossenen Volumen erschweren Reflexionen neben Signalrauschen und der Frequenzlimitierung der GHATT die Laufzeitbestimmung. Es ergibt sich somit aus (1) ein Multipfad-Problem, bei dem sich das Empfangssignal aus der additiven Überlagerung von $k$ Sendesignalen mit individuellen Zeitverzögerungen $\tau_{j}$ und Dämpfungsfaktoren $\alpha_{j}$, sowie einem additiven Rauschanteil $w$ zusammensetzt:

$$
x(t)=\sum_{j=1}^{k} \alpha_{j} \cdot s\left(t-\tau_{j}\right)+w(t)
$$

Das Multipfad-Modell aus (2) kann als Faltung des Sendesignals mit der Raumimpulsantwort verallgemeinert werden:

$$
x(t)=s(t) * h+w(t)
$$

Da neben der Raumimpulsantwort $h$ auch die eingesetzte Messtechnik Einfluss auf das aufgezeichnete Signal hat (Wiedergabeelektronik $h_{\text {play }}$, Lautsprecher $h_{L S}$, Aufnahmeelektronik $h_{\text {rec }}$ ), ergibt sich aus (3):

$$
x(t)=s(t) * h_{\text {play }} * h_{L S} * h * h_{\text {rec }}+w
$$

Um die für die Laufzeitbestimmung relevante Raumimpulsantwort $h \mathrm{zu}$ isolieren, werden in dieser Arbeit das Sendesignal $s$ und die systembedingten Signaleinflüsse in (4) durch $\tilde{s}$ substituiert:

mit

$$
x(t)=\tilde{s}(t) * h+w
$$

$$
\tilde{s}(t)=s(t) * h_{\text {play }} * h_{L S} * h_{\text {rec }}=s(t) * h_{\text {anecho }}
$$

Voraussetzung für die Anwendung ist eine Vorab-Bestimmung der reflexionsarmen Impulsantwort $h_{\text {anecho }}$, welche die raumunabhängigen, systembedingten Verzerrungen wiedergibt.

Da es bei dem Multipfad-Problem (3) bzw. (5) zu einer zeitlichen Überlagerung des Sendesignals und dem Signalrauschen kommt, scheitern korrelationsbasierte Verfahren häufig bei der Laufzeitbestimmung [5]. Die Ursache hierfür liegt in der Tatsache, dass die Überlagerung durch Interferenzen dazu führt, dass Korrelationsmaxima beeinflusst werden.

Der von Elfering et al. [6] beschriebene und in dieser Arbeit angewandte Ansatz zur Laufzeitbestimmung basiert auf der Approximation der Raumimpulsantwort $\hat{h}$ mittels dünnbesetzter Dekonvolution. Die einzelnen Impulse dieser Raumimpulsantwort entsprechen dabei dem direkten Schall respektive den reflektierten Schallanteilen. Der erste Impuls repräsentiert stets den akustisch kürzesten Pfad und ist daher für die Laufzeitbestimmung entscheidend.
Mathematisch ist das Ziel der dünnbesetzten Dekonvolution, die Abstandsquadrate zwischen dem Empfangssignal $s$ und der rekonstruierten Annäherung $\hat{h} * s$ zu reduzieren und dabei die Anzahl der Einträge in $\hat{h}$, welche nicht Null sind auf ein Maximalwert $\hat{k}$ zu begrenzen. Es ergibt sich somit folgendes Optimierungsproblem:

$$
\underset{\hat{h}}{\operatorname{argmin}}\|x-\tilde{s} * \hat{h}\|_{2} \text { s.t. }\|\hat{h}\|_{0} \leq \hat{k}
$$

Vor diesem Hintergrund wurden der Einsatz dünnbesetzter Dekonvolutionsansätze und optimierter Signale unter kontrollierten Bedingungen numerisch erprobt. Durch die Beschränkung auf den unterkritischen Frequenzbereich und durch den Frequenzbereich üblicher Unterwasserlautsprecher ergibt sich bei der GHATT ein praktischer Frequenzbereich von $500 \ldots 4000 \mathrm{~Hz}$. Zur Pulskompression und zur Verringerung der zeitlichen Überlappung des Direktschalls mit den Reflexionen verwendet die vorgestellte Methode kurze Signale mit einer linear modulierten Frequenz. Für eine zuverlässige Dekonvolution gilt es, die Autokorrelation zu disambiguieren, d.h. lokale Nebenmaxima zu reduzieren. Einerseits kann dies durch eine Verlängerung der Signale erreicht werden, allerdings führen längere Signale auch zu einer Steigerung der zeitlichen Signalüberlappung. Alternativ lassen sich mehrere kurze Signale einsetzen, sodass der direkte Schall weniger mit Reflexionen überlappt. [6]

\section{Numerische Erprobung}

Zur zielgerichteten Erprobung der Laufzeitbestimmung mittels dünnbesetzter Dekonvolution eignen sich numerische Verfahren, bei denen Parameter wie das SignalRausch-Verhältnis (SNR) oder die zugrundeliegende wahre Raumimpulsantwort bekannt sind und unabhängig voneinander variiert werden können.

Hierzu werden die Empfangssignale $x$ entsprechend (3) aus einem bekannten Sendesignal $s$ einer Raumimpulsantwort $h$ mit arbiträrer dünnbesetzter Struktur sowie additivem weißen Rauschen $w$ artifiziell erzeugt. Diese Empfangssignale lassen sich anschließend mittels dünnbesetzter Dekonvolution bezüglich ihrer Laufzeit auswerten.

Ein Beispiel eines solchen Signals und dessen Dekonvolution ist in Abb. 3 abgebildet. In Schwarz wird dabei das Sendesignal, die zugrundeliegende Raumimpulsantwort und das sich daraus ergebende Empfangssignal mit einem SNR $=3 \mathrm{~dB}$ dargestellt. Die zugrundeliegende Raumimpulsantwort wurde im gezeigten Beispiel mit einer Kardinalität $\|h\|_{0}=k=6$ generiert. In Rot sind die Ergebnisse des Optimierungsproblems (6) für $\hat{h}$ sowie dem approximierten Empfangssignal $\hat{h} * s$ dargestellt. Für die Laufzeitbestimmung ist der Zeitpunkt des ersten rekonstruierten Impulses entscheidend. Die weiteren Impulse spiegeln die Erstreflexionen wieder. Deren Rekonstruktion ist lediglich erforderlich, um die Lage des relevanten ersten Impuls zuverlässig und präzise bestimmen zu können.

Abb. 3 zeigt, dass das angenäherte Empfangssignal gut mit dem wahren Empfangssignal übereinstimmt. Auch das approximierte Ergebnis der Raumimpulsantwort und damit 
ihr für die Laufzeitbestimmung relevanter erster Impuls zeigen gute Übereinstimmung mit der wahren Raumimpulsantwort. Das Verfahren eignet sich in diesem Fall somit ideal für die Laufzeitbestimmung.
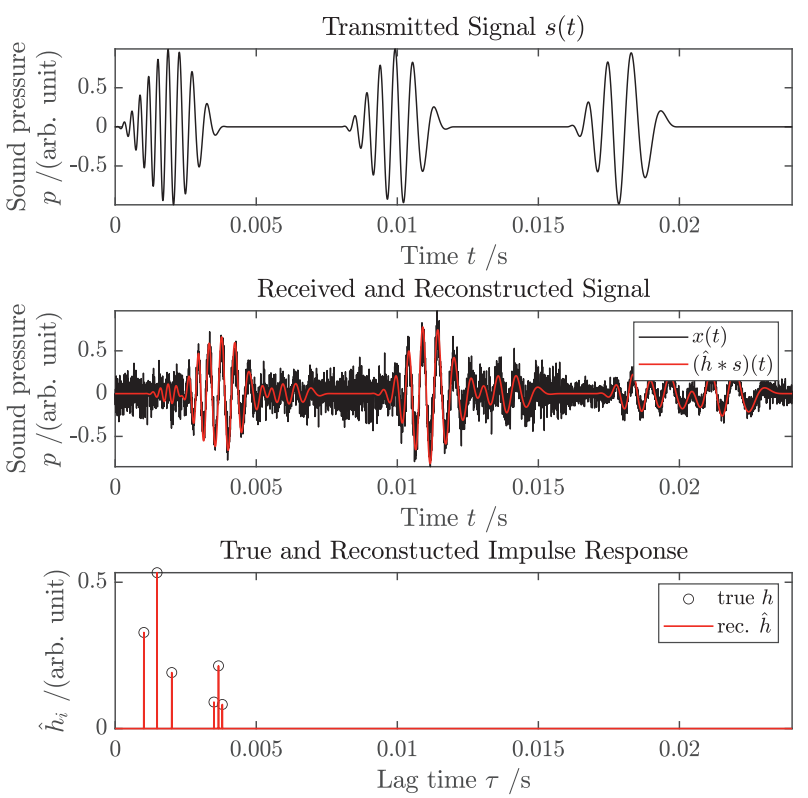

Abb. 3: Beispiel einer Signal-Dekonvolution mit dem gesendeten Signal $s$, dem simulierten Empfangssignal $x$ $(\mathrm{SNR}=3 \mathrm{~dB})$, der rekonstruierten und wahren Raumimpulsantwort $\hat{h}$ bzw. $h$ sowie dem daraus resultierenden Rekonstruktionsempfangssignal $\hat{h} * s$; Die Laufzeit ergibt sich aus dem ersten Peak der Impulsantwort.

Bemerkenswert an dem Empfangssignal in Abb. 3 ist, dass durch destruktive Interferenz des Direktschalls mit den ersten Reflexionen der Signalverlauf stark vom Sendesignal abweicht. Dies veranschaulicht, warum korrelationsbasierte Verfahren sich häufig als ungeeignet für verrauschte Mehrpfadprobleme erweisen.

Die statistische Auswertung von verschiedenen artifiziellen Empfangssignalen mit unterschiedlicher Raumimpulsantwort und SNR > $0 \mathrm{~dB}$ zeigen, dass die Raumimpulsantwort zuverlässig auch bei dicht aufeinanderfolgenden Impulsen und stark verrauschten Signalen rekonstruiert werden kann [6].

\section{Praktische Erprobung}

Neben der numerischen Erprobung des GHATT-Systems und der Laufzeitbestimmung ist insbesondere die praktische Erprobung unter kontrollierten Randbedingungen essenziell. Zu diesem Zweck wurde ein Laborprüfstand mit einem Volumen von $3 \mathrm{~m}^{3}$ aufgebaut (Abb. 4). Dieser ist optisch zugänglich und verfügt über eine variable Begasung am Behälterboden, sodass sich verschiedene Gasgehaltsverteilungen einstellen lassen. Für die akustischen Messungen werden jeweils bis zu acht Unterwasserlautsprecher und Hydrophone eingesetzt. Die Empfangssignale werden über ein Audiointerface aufgezeichnet. Die Lautsprecher lassen sich über einen elektronischen Umschalter einzeln für den Sendebetrieb ansteuern.

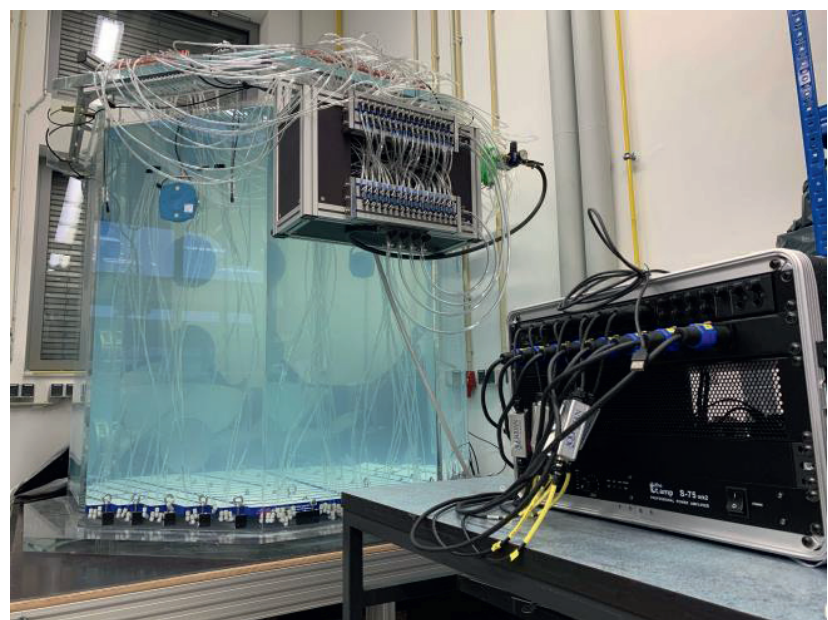

Abb. 4: Laborprüfstand für die Verfahrenserprobung, bestehend aus einem achteckigen Acrylglas-Behälter mit variabler Begasung, acht Unterwasserlautsprechern, acht Hydrophonen, einem Audiointerface und Signalumschalter für den Lautsprecherbetrieb

Zur praktischen Erprobung der Laufzeitbestimmung wurde einer der Lautsprecher wandnah im Prüfstand positioniert. Acht Hydrophone wurden analog zu Abb. 2 in derselben Höhe auf dem Umfang verteilt platziert. Der Lautsprecher wurde mit dem Sendesignal gespeist und die Empfangssignale der Hydrophone parallel aufgezeichnet und anschließend ausgewertet (Abb. 5).

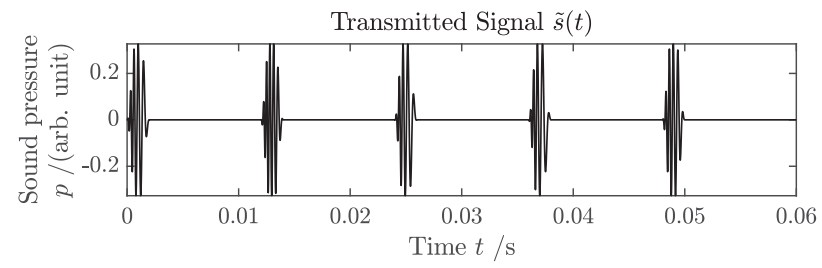

Received Signal
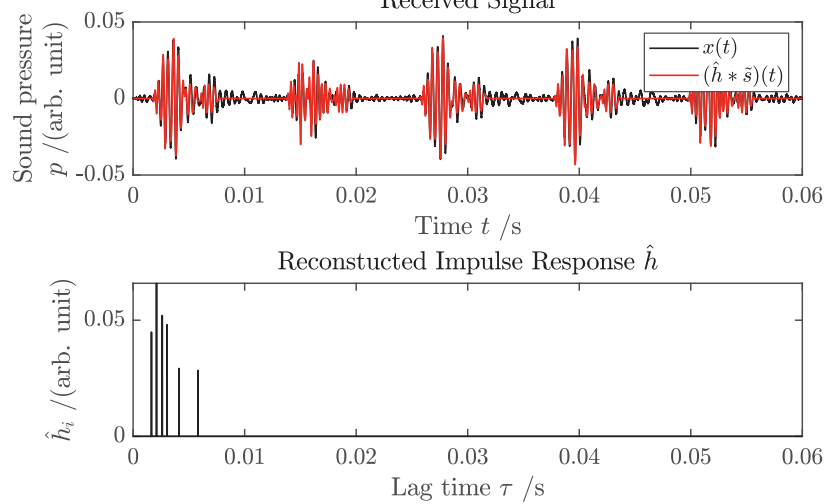

Abb. 5: Beispiel einer Signal-Dekonvolution mit dem gesendeten Signal $\hat{s}$, dem gemessenen Empfangssignal $x$, der rekonstruierten Raumimpulsantwort $\hat{h}$ und dem daraus resultierenden Rekonstruktionsempfangssignal $\hat{h} * s$; Die Laufzeit ergibt sich aus dem ersten Peak der Impulsantwort. 
Abb. 5 zeigt ein Beispiel der Dekonvolution eines aufgezeichneten Signals. Im gezeigten Beispiel wurde ein Signal bestehend aus fünf frequenzmodellierten Signalanteilen eingesetzt. Die rekonstruierte Raumimpulsantwort wurde mit $\hat{k}=6$ ermittelt. Dabei sollte $\hat{k}$ so gewählt werden, dass sich Erstreflexionen und der direkte Schallanteil abbilden lassen. Hierbei erwiesen sich Werte zwischen 4 und 8 als gute Praxis.

Der rekonstruierte Direktschall und die Erstreflexionen in Abb. 5 erscheinen für den betrachteten Behälter plausibel. Eine umfassende Validierung des Verfahrens ist jedoch noch nicht abgeschlossen. Die bisherigen Untersuchungen unterstützen jedoch bereits grundsätzlich die Erkenntnisse der numerischen Erprobung zur Eignung dieses Verfahrens zur Laufzeitbestimmung.

\section{Zusammenfassung und Ausblick}

Durch die beschriebene Methode lassen sich Laufzeiten niederfrequenter akustischer Signale in reflexionsreicher und verrauschter Umgebung bestimmen. Die bisher durchgeführten numerischen und labortechnischen Untersuchungen zum Einsatz dünnbesetzter Dekonvolutionsalgorithmen zur Laufzeitbestimmung in GHATT-Systemen zeigen bereits vielversprechende Ergebnisse, welche für deren Einsatz sprechen. Die ermittelten Laufzeiten können als Teil der GHATT-Signalauswertung für die Rekonstruktion von Schallgeschwindigkeitsverteilungen respektive Gasgehaltsverteilungen in Mehrphasensystemen dienen.

Für die sichere Anwendung gilt es jedoch zunächst, die praktischen Untersuchungen fortzusetzen und die Ergebnisse zu validieren. Nach Abschluss der Laboruntersuchung ist die großskalige Erprobung geplant. Mittelfristig soll mit der GHATT ein Verfahren entstehen, welches als Analysewerkzeug zur Optimierung der Anlagentechnik sowie zur Überwachung in den Bereichen der Verfahrenstechnik, Pharma- und Chemieindustrie sowie der Wasseraufbereitung oder der Biogasproduktion eingesetzt werden kann.

\section{Literatur}

[1] M. Elfering, D. Borgmann, H. Czajka, H.-A. Jantzen, $\mathrm{S}$. Annas und U. Janoske, „Introduction to an acoustic tomography system for the determination of the gas holdup" in MikroSystemTechnik Kongress 2019, 1 Aufl., Berlin: VDE VERLAG, 2019, S. 219221.

[2] M. Elfering, H. Czajka, S. Annas und H.-A. Jantzen, "Messanordnung und Verfahren zum Bestimmen einer Gasgehaltsverteilung," DE 102019128230 A1. DE 102019128230, Apr 22, 2021.

[3] A. B. Wood, A textbook of sound: Being an account of the physics of vibrations with special reference to recent theoretical and technical developments, 1. Aufl. The Macmillan company, 1930.

[4] S. Temkin, Suspension Acoustics. Cambridge: Cambridge University Press, 2005.

[5] R. Lee, M.-S. Kang, B.-H. Kim, K.-H. Park, S. Q. Lee und H.-M. Park, „Sound Source Localization
Based on GCC-PHAT With Diffuseness Mask in Noisy and Reverberant Environments", IEEE Access, Jg. 8, S. 7373-7382, 2020, doi: 10.1109/ACCESS.2019.2963768.

[6] M. Elfering, S. Annas, H.-A. Jantzen und U. Janoske, „Method for Time-of-Flight Estimation of Low Frequency Acoustic Signals in Reverberant and Noisy Environment with Sparse Impulse Response“, Meas. Sci. Technol., in Review.

\section{Danksagung}

Diese Arbeit wurde gefördert vom Bundesministerium für Bildung und Forschung (FKZ: 16ES0984).

GEFÖRDERT VOM

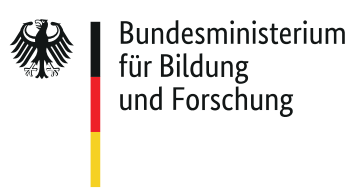

